on a bank holiday from a couple who were expressing suicidal thoughts and intentions. The volunteer was able to listen to their distress, contact the local crisis team and ensure they had access to the appropriate services to help them cope with their mental health needs. Another caller rang to talk through their experience of loss and grief following the death of a family member. They didn't have a close support network and needed a listening ear to help with their grief.

Impact It's clear the helpline provided a vital service during very stressful times for many needing a listening ear at any time of day or night. The helpline will remain part of Mountbatten's clinical services.

\section{P-56 COMMUNITY RESPONSE DURING THE COVID-19 PANDEMIC}

Gemma Allen, Claire Towns. The Mary Stevens Hospice, Stourbridge, UK

\subsection{6/spcare-2021-Hospice.74}

Background The Advice Hub launched on 15 April 2020 as an immediate community response during COVID-19, increasing capacity and providing community support. The aim was to build community capability and establish an Advice Hub for people with palliative care needs, care homes, health care professionals and members of the public.

Methods Emergency response funding was received from commissioners. The hospice converted a building into an Advice Hub with equipment, resources, and a wobble room, with staff available seven days a week providing advice and support. An online platform was developed with downloadable resources and information including:

- Advice Hub information.

- Caring for someone dying at home - advice for carers.

- Bereavement.

- Information for health care professionals.

- Advance care planning.

- Guidance for care homes.

- Palliative care for people with intellectual disabilities and COVID-19 information.

- Death and dying.

- Community support.

- Supporting someone living with dementia.

Results Initial evaluations indicated contact from care homes $(51 \%)$, carers $(26 \%)$, members of the public $(18 \%)$ and health care professionals (5\%). Data demonstrated contact related to practical advice (47\%), symptom control (18\%), signposting (14\%) bereavement (9\%), crisis intervention (4\%), advance care planning (4\%), and other (4\%). $13 \%$ of people received follow up contact. $34 \%$ of website users accessed the online resource. Between April and June 2020 the Advice Hub provided 440 hours of telephone support, with five admissions to the hospice.

Conclusion With an aging population and more people living with long term health conditions and co-morbidities, people requiring support during COVID-19 has increased. With calls from friends, neighbours, and family members it confirms that end of life care is everyone's business, with each community prepared to help (National Palliative and End of Life Care Partnership, 2021).

\section{P-57 'MAINTAINING EMOTIONAL BONDS DURING COVID-19 PANDEMIC'}

Debby Veigas, M Sandra Wild, Sarah Cook. Wakefield Hospice, Wakefield, UK

10.1136/spcare-2021-Hospice.75

Background Visiting restrictions have been challenging, fewer interactions increase anxiety and isolation (Mansfield, Mathur, Tazare, et al., 2021). Finding ways to maintain emotional bonds has been crucial to hospice care (Aghaei, Vanaki, Mohammadi, 2020; Mental Health Safety Improvement Team, [Royal College of Psychiatrists]) to help maintain connections (Azoulay, Kentish-Barnes, 2020). Using recordable cards and albums has had a positive impact on our service users, their family and on hospice staff.

Aims To maintain emotional connections between those receiving hospice care and their family given the impact of COVID19. To elucidate the experiences of those using recordable cards and albums, those who received them and hospice staff who supported their use.

Methods In 2018/19 the Hospice Admiral Nurse (HAN) started the use of recordable albums as part of life story work. When the pandemic hit the hospice introduced recordable cards and provided them free for all service users using grant funding and the HAN continued to support the community in their use, finding carers whose loved one had transitioned into a care home particularly benefitted. An evaluation form was attached to each card or album given out, with a good return rate.

Results People receiving inpatient hospice care preferred the recordable cards due to fatigue (age range 57-89). Evaluation received 5 out of 5 rating -'Comforting', 'Meaningful' and 'Worthwhile' were the most used words -with some family members crying with joy at receiving a recorded card. Albums were mostly used in the community and given a 5 rating; carers described the comfort of sending their album into the care home which not only maintained an emotional connection but also allowed staff to get to know the person quickly.

Conclusions Evaluations demonstrated the emotional impact using recordable cards and albums had on hospice service users and their family. Hospice staff found being alongside, supporting people recording messages improved their own mental wellbeing and job satisfaction.

\section{P-58 ONLINE DELIVERY OF MINDFULNESS AND MEDITATION PSYCHOLOGICAL SUPPORT GROUP}

Abigail Naylor. Health and Wellbeing Centre, North London Hospice, London, UK

\subsection{6/spcare-2021-Hospice.76}

Background Social connectedness is a strong predictor of mental health (Saeri, Cruwys, Barlow, et al., 2018), yet the COVID-19 pandemic and ensuing government-ordered lockdowns forced the UK population to limit their social contact. This presented a challenge to the provision of in-person psychological services to the clinically vulnerable patients of the Health and Wellbeing Centre at North London Hospice. Online social contact has been associated with positive psychological outcomes (Grieve, Indian, Witteveen, et al., 2013) and online mindfulness-based interventions have shown to reduce psychological distress (Ma, She, Siu, et al., 2018). After a 
number of patients reported a willingness to try an online group, we trialled a six-week online Mindfulness and Meditation group.

Aim(s) To discuss the principles of mindfulness and how it can benefit those living with life-limiting illnesses; deliver tangible experience of mindfulness practice; and provide social connectedness to patients with symptoms of anxiety shielding in their homes.

Methods November - December 2020: course scoping and planning; December 2020 - January 2021: patient recruitment; 27 January - 3 March 2021: course delivered via presentation slides on Zoom and weekly homework tasks; March - April 2021: evaluation and service user feedback. Outcome measures: number of attendees, service user feedback.

Results Attendee numbers: 11 patients signed up with between five and eight attending each session. Six patients provided feedback: 100\% reported the course helped them a) feel less stressed and overwhelmed, b) change the way they view their thoughts, and c) change their behaviours. $83 \%$ were either 'satisfied' or 'very satisfied' with the course.

Conclusions The online Mindfulness and Meditation group had good and regular attendance, and service users indicated that it contributed to positive changes in their mental health. The course will run online again in July 2021, this time with pre- and post-questionnaires to assess the reduction in symptoms of anxiety more robustly.

\section{P-59 GROWING CONNECTIONS}

Lucy Heaps. Garden House Hospice Care, Letchworth Garden City, UK

10.1136/spcare-2021-Hospice.77

Background In March 2020, due to COVID-19 the Social and Therapeutic Horticulture (STH) group in day services had to close. The hospice occupational therapist (OT) wanted to support the group to stay connected to nature and each other, so decided to introduce an interactive monthly newsletter.

Aims To encourage and support patients and volunteers to connect with nature (and each other) to support their mental health and sense of wellbeing through a period of shielding and isolation.

Approach

- April 2020 - monthly newsletters were posted to patients and volunteers.

- June 2021 - reviewed and agreed plans to relaunch the STH face-to-face groups (following the social distancing and PPE requirements).

- August 2021 - face-to-face 'wellbeing through nature' sessions to start and newsletters to continue.

Results 32 people have accessed the monthly newsletter, whereas the face-to-face session only had six per session (on average). It has reached patients who had been unable to attend sessions at the centre due to logistics and care needs. It has enabled new patients to connect and benefit. It has kept people connected as they shared their news and stories together in the 'grapevine' section of the newsletter. The OT has inspired good practice for other health professionals.

Conclusion The newsletter has proven to be a fantastic medium for patients and volunteers to stay connected to the hospice and their peers through the pandemic. It has gathered a great deal of positive feedback from patients and families/ carers alike. It has enabled the service to demonstrate that even through the pandemic we have been able to keep to our hospice core values. The newsletter will continue post COVID-19. It has enabled our patients to connect in a virtual way. We now look forward to restarting our face-to-face STH sessions in our day service.

\section{P-60 COMPTON VS COVID: STEPPING UP}

Beverley Clarke, Tricia Evans, Jenny Warren, Angela Matthews, James Elliott. Compton Care, Wolverhampton, UK

\subsection{6/spcare-2021-Hospice.78}

Background and Aims In March 2020 our hospice was faced with an unprecedented challenge. The COVID-19 pandemic affected every aspect of our work, from patient care to fundraising, but proactive cooperation with local services enabled us to 'Step Up' our support for patients and families.

Method and Results The 'Stepping Up' appeal found innovative ways to raise funds and engage with the public, from virtual events to gathering messages of support for our hardworking staff. Our Compassionate Communities team launched a telephone befriending service and virtual bereavement information hubs, reaching out to those isolated by the pandemic. Our inpatient unit expanded to better facilitate admissions from local hospitals and the community, overseen by a dedicated flow co-ordinator. The team was rapidly upskilled in managing more acutely unwell patients, including those with COVID-19, and challenging situations such as withdrawal of NIV.

Our Living Well Centre, temporarily closed due to the pandemic, was transformed into a nurse-led unit for COVID-19positive patients awaiting care home placement. As well as being redeployed, our Living Well nurses embraced new technology to support their patients remotely. Our community team provided additional support to local district nursing teams, taking over personal care for some of their more complex palliative patients. Training in procedures such as catheter insertion and venepuncture was also expedited. Working collaboratively with NHS colleagues we were able to manage more complex medical situations at home, reducing the need for inpatient admissions. Video consultations and a professional support line allowed the medical team to become more responsive.

Conclusion Despite the many challenges, we have taken the opportunity to improve continuity of care and build better working relationships within our organisation. Cooperation with external partners, a keystone of good palliative care, has been vital to our ability to respond and adapt, and we hope to see these positive changes continue.

\section{P-61 ST LEONARD'S HOSPICE COVID-19 RESPONSE - COORDINATION AND RESPONSE}

Jenny Brandom. St Leonard's Hospice, York, UK

\subsection{6/spcare-2021-Hospice. 79}

Background St Leonard's Hospice were approached by the local health and care system to lead the response and co-ordination of care for those people requiring end-of-life care in 